\title{
Sobre condiciones suficientes de estabilidad para sistemas mecánicos sometidos a fuerzas dependientes de la velocidad por un método variacional
}

RAFAEL RODRIGUEZ REBOLLO

$070-10$

Dr. Ingeniero de Caminos, Canales y Puertos

sinopsis

\begin{abstract}
Consideramos la estabilidad de un estado de un sistema mecánico, discreto o continuo, sometido a fuerzas generalizadas dependientes o no de la velocidad y cuyas ecuaciones variacionales de movimiento, para pequeñas perturbaciones, son ecuaciones diferenciales de segundo orden respecto del tiempo y de una estructura formal frecuente en diversos problemas de estabilidad de medios continuos y de sistemas de particulas y de sólidos rígidos. Se obtienen criterios de estabilidad y de estabilidad asintótica y se generalizan resultados conocidos, considerando los correspondientes problemas de autovalores para soluciones de tipo exponencial respecto del tiempo.
\end{abstract}

\section{Introducción}

En un trabajo anterior [1], se consideró la estabilidad de estados de ciertos sistemas discretos o continuos, de forma unificada, mediante la aplicación del método directo o segundo método de Liapunov a la ecuación variacional de movimiento que obedecian, escrita en forma operacional. Intervenian fuerzas generalizadas dependientes o no de la velocidad y se consideraban soluciones exponenciales en el tiempo. Respecto a la utilización de estas soluciones, citamos entre otros muchos autores, a Leipholtz [2].

En el presente trabajo, para la misma ecuación operacional, consideramos el problema de autovalores, correspondiente a aquellas soluciones, por un método variacional y obtenemos criterios de estabilidad.

Este método resulta ser de más utilidad para las aplicaciones prácticas.

\section{Formulación general}

Consideremos las ecuaciones variacionales de movimiento, para pequeñas perturbaciones, respecto de una configuración $C$ de la que se estudian las propiedades de estabilidad. De forma general, se escriben como sigue [1]:

$$
\hat{F} \vec{u} \equiv \ddot{\vec{u}}+2 \hat{E} * \dot{\vec{u}}+\hat{D} \vec{u}=\overrightarrow{0}
$$

La configuración $C$ corresponde a $\vec{u}(x, t)=\overrightarrow{0}$ donde $\vec{x}$ representa el vector posición en $V$, volumen material en $C$.

$\hat{F}, \hat{E}$, y $\hat{D}$ son operadores lineales diferenciales sobre $\vec{x}$, con coeficientes reales constantes, o bien matrices, con elementos reales. Cuando $\hat{E}=\hat{E}^{s}$, siendo $\hat{E}^{s}$ un operador hermítico (matriz simétrica) positivo, el término 2 $\hat{E}^{s} \vec{u}$ constituye un caso generalizado de fuerzas disipativas y cuando $\hat{E}=$ $=E^{a}$, donde $E^{a}$ es un operador antihermítico (matriz antisimétrica), constituyen fuerzas giroscópicas generalizadas. $\hat{D}=\hat{D^{s}}$, operador hermitico (matriz simétrica), corresponde a fuerzas derivables de una función potencial.

Consideramos las soluciones de la forma $\vec{u}=\vec{\eta}=\vec{f}(\vec{x}) e^{(i \alpha-\beta) t}$, donde $\alpha$ y $\beta$ reales, $\beta \geqslant 0$ y $\vec{f}$ es un vector columna, cuyas componentes son funciones de $\vec{x}$. Constituyen un espacio $P$, en el que definimos el producto interno:

$$
(\vec{\eta}, \vec{\xi})=\int_{v(t)} \vec{\eta}^{*} \cdot \vec{\xi} d V, \vec{\eta}, \vec{\xi} \in P
$$


donde el asterisco indica "complejo conjugado», siendo la integral finita. La norma de $\vec{\eta} \in P$ es:

$$
(\vec{\eta}, \vec{\eta})=\int_{v(t)} \vec{\eta}^{*} \cdot \vec{\eta} d V
$$

\section{Condiciones suficientes de estabilidad}

Sea la ecuación operacional (1). En el caso general, $\hat{E}=\hat{E}^{\mathrm{s}}+\hat{E}^{a}$ y $\hat{D}=\hat{D}^{\mathrm{s}}+$ $+\hat{D}^{a}$, donde $\hat{E}^{s}$ y $\hat{D}^{s}$ son operadores hermíticos y $\hat{E}^{a}$ y $\hat{D}^{a}$, operadores antihermíticos. Sustituyendo $\vec{u}=\vec{\eta}$ en (1), obtenemos:

$$
\ddot{\vec{\eta}}=-\left(\hat{G}^{s}+\hat{H}^{a}\right) \vec{\eta}=-\hat{L \eta}=-\left[\left(\alpha^{2}-\beta^{2}\right)+2 \alpha \beta i\right] \vec{\eta}
$$

donde

$$
\begin{aligned}
& \hat{G}^{s}=\hat{G}_{1}^{s}+\hat{G}_{2}^{s}, \hat{H}^{\mathrm{a}}=\hat{H}_{1}^{\mathrm{a}}+\hat{H}_{2}^{\mathrm{a}} \\
& \hat{G}_{1}^{s}=D^{s}-2 \beta \hat{E}^{s} \\
& \hat{G}_{2}^{s}=2 \alpha i \hat{E}^{\mathrm{a}} \\
& \hat{H}_{1}^{\mathrm{a}}=\hat{D}^{\mathrm{a}}-2 \beta \hat{E}^{\mathrm{a}} \\
& \hat{H}_{2}^{\mathrm{a}}=2 \alpha i \hat{E}^{s}
\end{aligned}
$$

Fundamentalmente, consideramos dos casos:

I. Suponemos que las fuerzas que actúan sobre el sistema son conservativas y giroscópicas: $\hat{E}=\hat{E}^{a}$ y $\hat{D}=\hat{D}^{s}$.

Consideramos las soluciones $\vec{u}=\vec{\eta}$ para $\beta=0$, $\alpha$ real.

Tenemos:

$$
\widehat{F \eta} \equiv\left(\hat{D}^{s}+2 \alpha i \hat{E}^{\mathbf{a}}-\alpha^{2} \hat{\eta}\right) \vec{\eta}=\left(\hat{G}^{s}-\alpha^{2} \hat{T}\right) \vec{\eta}
$$

donde $\hat{\imath}$ es el operador unidad. Lo que nos prueba que $\hat{F}$ es un operador hermítico y tiene las mismas funciones propias que $G^{s}$. Los valores propios verifican la igualdad:

$$
\lambda_{F}=\lambda_{G}-\omega^{2}
$$

Para $\vec{\eta}$ solución de $\overrightarrow{F u}=\overrightarrow{0}$ :

$$
\hat{G} \vec{\eta}=\omega^{\overrightarrow{2} \eta}
$$

$\vec{\eta}$ es función propia de $\hat{G}^{s}$, cuyos autovalores son positivos. Por tanto, para $\hat{E}^{s}=\hat{D}^{a}=\hat{0}, G^{s}$ es un operador hermítico positivo para las $\vec{\eta}$, soluciones de $\hat{F} \vec{u}=\overrightarrow{0}$.

Esta condición respecto a $\hat{G}^{s}$ es equivalente al criterio obtenido en [1] por el método directo de Liapunov.

Efectivamente, $\left(\vec{\eta}, \hat{G}^{\vec{\eta}} \vec{\eta}\right) \geqslant c(\vec{\eta}, \vec{\eta}), c>0$, para todo posible $\vec{\eta}$.

La aplicación del criterio requiere la obtención de los valores propios $\lambda_{F}$. Sabemos que el problema de encontrar las soluciones de:

$$
\hat{F}[\vec{\eta}(\alpha)]=\vec{\varphi}(\alpha)
$$

donde $\hat{F}$ es un operador hermítico positivo, se resuelve calculando los extremos de la funcional:

$$
\Phi=(\vec{\eta}, \overrightarrow{F \eta})-2(\vec{\eta}, \vec{\varphi})
$$

La solución es la que hace $\Phi$ mínimo, siendo (.) la norma en el espacio que se considera.

En el caso que nos ocupa, las $\lambda_{F}$ se obtienen calculando los extremos de la funcional:

$$
\overrightarrow{(\eta}, \overrightarrow{F \eta})
$$


Basta considerar la funcional:

$$
M=\frac{\overrightarrow{(\eta}, \overrightarrow{F \eta})}{(\eta, \vec{\eta})}
$$

y las funciones propias $\vec{\eta}$ y los valores propios $\lambda=\lambda_{F}$ de $\hat{F}$ se obtienen de $\delta M=0$.

Podemos escribir:

$$
M=\frac{\overrightarrow{(\eta}, \overrightarrow{F \eta})}{(\vec{\eta}, \vec{\eta})}=\frac{\left.\overrightarrow{(\eta}, \hat{G}^{s} \vec{\eta}\right)}{\overrightarrow{(\eta}, \vec{\eta})}-\omega^{2}
$$

Los valores de $M$ tales que $\delta M=0$, para $\overrightarrow{\delta \eta}=\overrightarrow{\delta f} \cdot e^{i \omega t}$ son los autovalores $\dot{\lambda}_{F}$

Si hacemos $\lambda_{F}=\lambda_{G}-\omega^{2}=0$, los valores de $\omega$ soluciones de esta ecuación han de ser reales. Esta es la condición necesaria de estabilidad.

La condición $\lambda_{G}=\omega^{2}$ resume el criterio obtenido por el método energético.

La solución general de $\overrightarrow{F u}=\dot{\overrightarrow{0}}$ será la combinación lineal:

$$
\vec{\eta}=\sum_{k} \vec{f}_{k}(\vec{x}) e^{ \pm i \omega_{k t}}
$$

Para sistemas continuos, el número de valores $\omega^{2}$ que obtengamos será infinito.

La solución general pertenece también al espacio funcional $P$. Obtenemos condiciones suficientes.

Analizamos dos casos:

la. No existen fuerzas giroscópicas, $\hat{E}^{a}=\hat{0}$

donde $\lambda_{G}$ no depende de $\omega$.

$$
M=\frac{\left.\overrightarrow{(\eta}, \overrightarrow{D^{s} \vec{\eta}}\right)}{(\vec{\eta}, \vec{\eta})}-\omega^{2}
$$

En el plano $\left(\omega, \lambda_{F}\right)$ la función $\lambda_{F}$ tiene un máximo para $\omega=0$, que marca la condición límite de estabilidad: $\frac{\partial \lambda_{F}}{\partial \omega}=-2 \omega=0 \Rightarrow \omega=0,\left.\frac{\partial^{2} \lambda_{F}}{\partial \omega^{2}}\right|_{\omega=0}=-2<0$.

La condición de estabilidad se expresa de forma más simple considerando el $\lambda_{F}$ de valor más pequeño para $\omega=0$. Ha de ser, como condición suficiente:

$$
\text { menor }\left.\lambda_{F}\right|_{\omega=0}>0
$$

Suponemos que en el caso general el espectro de autovalores está acotado por un lado.

Ib. En el caso de existencia de fuerzas giroscópicas, $\lambda_{F}=\lambda_{G}-\omega^{2}$, con $\lambda_{G}$ función de $\omega$.

Cada función $\lambda_{F}$ ha de tener, como en el caso anterior, extremos a un lado (u otro) de $\lambda_{F}=0$ y ha de intersectar el eje $\lambda_{F}=0$.

Estos extremos no serán para $\omega=0$ en general, pues $\hat{G}=\hat{D}^{s}+2 \omega i \hat{E}^{a}-\omega^{2} \hat{I}$ puede depender de forma no lineal de $\omega$. En el caso particular de dependencia lineal, las condiciones de estabilidad se obtendrán de:

$$
-\omega^{2}+f(s) \omega+g(s)=0
$$

con $f^{2}(s)+4 g(s) \geqslant 0$, donde $f(s)$ y $g(s)$ son ciertas funciones de los parámetros $s$ del sistema.

Geométricamente, en el plano $\left(\omega, \lambda_{F}\right)$ obtenemos una serie de curvas $\lambda_{F}$ tales que $\lambda_{F} \rightarrow-\chi$ con $\omega \rightarrow \pm \chi$. Por tanto, condiciones suficientes de estabilidad se obtienen calculando:

$$
\frac{\partial \lambda_{F}}{\partial \omega}=0 \Rightarrow \omega=\omega^{\prime}
$$


y haciendo que para dichas $\omega^{\prime}$, las $\lambda_{F}$ sean positivas.

Si el espectro de autovalores es acotado, bastará expresar que el menor de dichos $\lambda_{F}$ sea positivo.

En el caso particular de dependencia lineal, las $\lambda_{F}$ son parábolas a un lado y otro del eje $\lambda_{F}$.

II. Caso general. Según (2):

$$
\vec{F} \equiv\left(\vec{G}^{s}+\vec{H}^{\mathrm{a}}-\omega^{2} \hat{\imath}\right) \vec{\eta}=\lambda_{F} \vec{\eta}=\left(\lambda_{F}^{r}+i \lambda_{F}^{i}\right) \vec{\eta}
$$

donde los superíndices «r» e «i». indican «real» e «imaginario», respectivamente.

Como antes, consideramos la funcional $M$, cuyos extremos son los autovalores $\lambda_{F}$.

Ha de ser:

$$
\lambda_{F}=\lambda_{G+H}-\omega^{2}=\lambda_{F}^{r}+i \lambda_{F}^{i}=0, \omega^{2}=\alpha^{2}-\beta^{2}+2 \alpha \beta i
$$

y por tanto:

$$
\begin{aligned}
& \lambda_{F}^{r}=0 \\
& \lambda_{F}^{i}=0
\end{aligned}
$$

para $\omega=\alpha+i \beta, \alpha$ y $\beta$ reales, $\beta \geqslant 0 . \beta>0$ corresponde a estabilidad asintótica.

Anteriormente considerábamos el plano $\left(\omega, \lambda_{F}\right)$, $\omega$ real. Ahora consideramos el triedro trirrectángulo $\left(\alpha, \beta, \lambda_{F}^{r}\right) \beta \geqslant 0$ y $\alpha$ reales. El plano $\left(\alpha, \lambda_{F}^{r}\right)$ marca el límite que separa las soluciones exponencialmente crecientes de las decrecientes. La condición $\lambda_{F}^{i}=0$ representa una curva en el plano $(\alpha, \beta)$.

La condición de estabilidad se expresa diciendo que $\lambda_{F}^{r}=0$ para $\omega=\alpha+i \beta$, $\alpha$ y $\beta$ reales, $\beta \geqslant 0$, tales que $\lambda_{F}^{i}=0$. Los valores $\beta>0$ corresponden a estabilidad asintótica.

Las condiciones límites que separan la inestabilidad de la estabilidad asintótica nos la da $\beta=0$. Si $\widehat{G}^{s}+\hat{H}^{a}$ contiene a $\alpha$, condición suficiente de estabilidad es:

$$
\text { Menor }\left.\lambda_{F}^{r}\right|_{\alpha=\alpha^{\prime}}>0, \alpha=\alpha^{\prime} \text { tal que }\left.\lambda_{F}^{i}\right|_{\alpha=\alpha^{\prime}}=0, \beta=0
$$

\section{Referencias}

[1] R. Rodriguez Rebollo: «Sobre condiciones suficientes de estabilidad para sistemas mecánicos sometidos a fuerzas dependientes de la velocidad por el método directo de Liapunov". Para ser publicado

[2] Leipholtz, H.: «Stability theory". New York, London: Academic Press (1970).

[3] Leipholtz, H.: «Uber das statiche kriterium bei nichtkonservativen stabilitäts problemen der Elastomechanik». Ingenieur Archiv 32, 214-220 (1963).

[4] Leipholtz, H.: «Uber ein kriterium für die Gültigkeit der statischen Methode zur Bestimmumg der knicklast von elastischen stäben unter nicht conservativer Belastung. Ingenieur Archiv 32, 286-296 (1963).

[5] Leipholtz, H.: «Uber den Einfluss der Dämpfung bei nicht conservativen stabilitäts problemen elastischer stäbe». Ing. Arch. 33, 308-321 (1964). 


\section{résumé}

A propos des conditions suffisantes de stabilité à l'aide de systèmes mécaniques soumis à des forces dépendantes de la vitesse a l'aide de la méthode directe de Liapunov.

Rafael Rodríguez Rebollo,

Dr. Ingénieur des Ponts et Chaussées

Considérons la stabilité d'un état d'un système mécanique, discret ou continu, soumis à des forces généralisées dépendantes ou non de la vitesse dont les équations variationnelles de mouvement, pour des petites perturbations, sont des équations différentielles de second ordre par rapport ay temps et d'une structure for melle fréquente en divers problèmes de stabilité de moyens continus et de systèmes de particules et de solides rigides.

On obtient des critères de stabilité et de stabilité asymptote selon Liapunov à l'aide de la méthode directe, en généralisant des résultats connus. Ce sont des conditions de stabilité conditionnelle pour des solutions de type exponentiel par rapport au temps.

A propos des conditions suffisantes de stabilité à l'aide de systèmes mécaniques soumis à des forces dépendantes de la vitesse à l'aide d'une méthode variationnelle.

Considérons la stabilité d'un état d'un système mécanique discret ou continu, soumis à des forces généralisées dépensoumis à des forces généralisées dépendantes ou non de la vitesse dont l'équation variationnelle de mouvement, pour des petites perturbations, sont des équations différentielles de second ordre par rapport au temps et d'un structure formelle fréquente en divers problèmes de stabilité de moyens continus et de systèmes de particules et de solides rigides.

On obtient de critères de stabilité et de stabilité asymptote et on généralise des résultats connus, considérents les problèmes correspondants d'autovaleurs pour des solutions de type exponentie par rapport au temps.

\section{summary}

On sufficient stability conditions for mechanical systems subject to speed-dependent stresses, by the direct Liapunov's method

\section{By Rafael Rodríguez Rebollo,}

Dr. in Civil Engineering

We are considering here the stability of a mechanical system state, either discrete or continuous, subject to overall stresses, whether or not dependent on speed, having variational movement equations for equations of the second degree in respect to time and with a formal structure, frequent in several problems of stability in continuous media and in systems of solid rigid particles.

Stability and asymptotic stability criteria are obtained after Liapunov, by the direct method, thus generalising known results. These are conditions of conditional stabiThese are conditions of conditional stabi-
lity for exponential type solutions in reslity for expone
pect to time.

On sufficient stability conditions for mechanical systems subject to speed-dependent stresses, by a variational method

We are considering here the stability of a mechanical system state, either discrete or continuous, subject to overall stresses, whether or not dependent on speed, having variational movement equations for small disturbances which are differential equations of the second degree in respect to time and with a formal structure frequent in several problems of stability in continuous media and system of solid rigid particles. Stability and asymptotic stability criteria are obtained, thus generalising known results, considering self-value problems for exponential type results in respect to time.

\section{zusammenfassung}

Ueber ausreichende bestaendigkeitsbedingungen fuer mechanische systeme, welche nach der direkten Liapunov-methode geschwindigkeitsabhaengigen belastungen ausgesetzt werden

Dr. Rafael Rodríguez Rebollo,

Bauingenieur

Wir betrachten die Beständigkeit eines Zustands eines diskreten oder kontinuierlichen mechanischen Systems, welches verallgemeinerten Belastungen in Abhängigkeit oder Unabhängigkeit von der Geschwindigkeit ausgesetzt werden, und dessen variablen Bewegungsgleichungen für kleine Störungen Differentialgleichungen zweiten Grades bezüglich der Zeit sind und einer formellen Struktur, welche bei verschiedenen Beständigkeitsproblemen kontinuierlicher Mittel und Teilchensystemen sowie starren Feststoffen häufig vorkommt.

Nach Liapunov werden Kriterien über die Standfestigkeit und asymptotische Stabilität durch die direkte Methode bei Verallgemeinerung der bekannten Ergebnisse erhalten. Es handelt sich um bedingte Beständigkeitsbedingungen für Lösungen darstellender Art im Hinblick auf die Zeit.

Ueber ausreichende bestaendigkeitsbedingungen fuer mechanische systeme, welche geschwindigkeitsabhaengigen belastungen nach einer variationsmethode ausgesetzt werden

Wir betrachten die Beständigkeit eines Zustandes eines diskreten oder kontinuierlichen Systems, welches verallgemeinerten Belastungen in Abhängigkeit oder Unabhängigkeit von der Geschwindigkeit ausgesetzt werden, dessen variablen Bewegungsgleichungen bezüglich der Zeit Differentialgleichungen zweiten Grades sind, und einer formellen Struktur welche bei verschiedenen Beständigwelche bei verschiedenen Beständigund Teilchensystemen sowie starren Feststoffen häufig vorkommt. Unter $\mathrm{Be}$ rücksichtigung der entsprechenden Selbstwertprobleme für Lösungen darsteSender Art im Hinblick auf die Zeit, werden Kriterien über die Standfestigkeit und asymptotische Stabilität erhalten und bekannte Ergebnisse verallgemeinert. 